\title{
Reduced frequency of knowledge of results enhances learning in persons with Parkinson's disease
}

\section{Suzete Chiviacowsky*, Tiago Campos and Marlos Rodrigues Domingues}

School of Physical Education, Federal University of Pelotas, Pelotas, Rio Grande do Sul, Brazil

\section{Edited by:}

Charles H. Shea, Texas A\&M

University, USA

\section{Reviewed by:}

Jeffrey T. Fairbrother, University of

Tennessee, USA

Beth Fisher, University of Southern

California, USA

*Correspondence:

Suzete Chiviacowsky, Escola Superior de Educação Física, Universidade

Federal de Pelotas, Rua Luís de

Camões, 625 - CEP 96055-630,

Pelotas, Rio Grande do Sul, Brazil.

e-mail: schivi@terra.com.br
Parkinson's disease (PD) is a progressive neurological disorder, known to cause a large number of motor and non-motor limitations. Research related to factors that affect motor control and learning in people with $\mathrm{PD}$ is still relatively limited. The purpose of this study was to compare the effects of different frequencies (100 versus 66\%) of knowledge of results (KR) on the learning of a motor skill with spatial demands in participants with PD. Twenty individuals with PD were randomly assigned to one of two groups. The $100 \%$ group received $\mathrm{KR}$ after each trial, while the $66 \%$ group received KR on two thirds of the trials. A linear positioning task with a spatial target was used. Participants carried out the task with the dominant hand while blindfolded. In the acquisition and retention phases, the goal was to position the cursor at a distance of $60 \mathrm{~cm}$ from the starting point. The hypothesis was that participants with PD, who practiced with a reduced $K R$ frequency, would demonstrate more effective learning than those who practiced with a $100 \% \mathrm{KR}$ frequency, similar to previous findings with adults without neurological disorders. The results showed differences between the groups in the retention phase (without KR): The $66 \% \mathrm{KR}$ group was more accurate and less variable in their performance than the $100 \% \mathrm{KR}$ group. Thus, reducing KR frequency can enhance motor learning in persons with $\mathrm{PD}$, similar to what has previously been found for unimpaired participants.

Keywords: motor learning, feedback, knowledge of results, Parkinson's disease

\section{INTRODUCTION}

Parkinson's disease (PD) is a progressive neurological disorder, known to cause a large number of motor problems, such as tremor at rest, rigidity, bradykinesia, and postural instability (Jankovic, 2008). The main cause of PD symptoms is the decrease in dopamine production, with subsequent disruption of normal motor cortex activity. As a consequence, people with PD present motor control deficits in planning, initiating and executing movements, resulting in prolonged reaction times and abnormal pre-movement EMG activity (Berardelli et al., 2001), as well as longer and more variable deceleration phases of aiming movements (Rand et al., 2000), compared with people without neurological disorders. Numerous studies have also shown that PD is also associated with perceptual deficits, including visual depth perception (Maschke et al., 2003) and kinesthetic sensitivity (Demirci et al., 1997; Adamovich et al., 2001; Contreras-Vidal and Gold, 2004).

Fortunately, recent studies have been indicating positive results for interventions aiming at challenging impaired systems in PD, promoting recovery instead of mere compensation, with the potential of reversing or delaying disease progression in this population (Tillerson et al., 2003; Herman et al., 2007; Petzinger et al., 2010). According to Hirsch and Farley (2009), oriented exercise may promote brain repair and reorganization (neuroplasticity) in people with PD, accompanied by behavioral recovery. Some studies using animals (Fisher et al., 2004; Petzinger et al., 2007), for example, have shown alterations in both dopaminergic and glutamatergic neurotransmission, by inducing activity-dependent (exercise) processes, mitigating the cortically driven hyper-excitability in the basal ganglia, characteristic of persons with PD. In addition, in a recent review of the literature, Nieuwboer et al. (2009) showed that, although people with PD did demonstrate slower learning rates than controls, their capability to learn motor skills was relatively preserved. Therefore, success of rehabilitation therapy depends, to a large extent, on the effectiveness of motor learning strategies (Abbruzzese et al., 2009).

An important practice variable known to improve motor learning in healthy populations is augmented or extrinsic feedback (Wulf et al., 2010b). Information regarding the pattern of movement used to perform the action (knowledge of performance, KP) and/or the outcome of the movement (knowledge of results, KR) is considered a powerful variable, capable of guiding the learner to the goal movement. Nevertheless, numerous studies with typical participants have demonstrated that, despite its benefits, augmented feedback can cause a dependency when provided in excess. Frequent extrinsic feedback is assumed to impede the processing of intrinsic feedback information, which is important for long-term retention of the goal movement (Salmoni et al., 1984; Schmidt, 1991).

The first studies showing detrimental effects of frequent feedback on motor learning used sequential spatial-timing tasks, and demonstrated superior results for reduced frequencies in the learning of a single task (Winstein and Schmidt, 1990) or different versions of the same task (generalized motor program learning; Wulf and Schmidt, 1989). Following these pioneering studies, several others have demonstrated the beneficial effects of reduced frequencies of KR for people of different developmental levels (children, young, and older adults) and for a wide range of tasks (for a review, see Wulf and Shea, 2004). 
Research related to factors that influence motor learning in people with Parkinson's disease is still relatively limited. Only a few studies have addressed the effects of augmented feedback in this population (e.g., Verschueren et al., 1997; Guadagnoli et al., 2002; Onla-or and Winstein, 2008). In the study by Verschueren et al. (1997), using a bimanual coordination task, participants were instructed to produce cyclical movements coincident with the beating of a electronic metronome, such that one complete movement cycle (flexion-extension) was performed with every beat, to facilitate the learning of a new spatio-temporal coordination pattern between the limbs (oscillation with a phase offset of $90^{\circ}$ between limbs). Augmented concurrent visual feedback was provided during all acquisition trials, with the aid of a computer monitor. The results, measured on a retention test after a 5-min interval, demonstrated a greater dependency of persons with PD on the augmented concurrent feedback presented during the trials. That is, performance deteriorated considerably when the extrinsic information was no longer available, in contrast to the behavior seen in a typical population.

In another experiment, Guadagnoli et al. (2002) reported an interaction between two different frequencies of KR, 100 and 20\%, in the learning of a timing task, in persons with PD and typical participants. In this study the task required participants, holding a stylus in their dominant hand, to perform pointing movements to a target, as accurately as possible and in a certain goal movement time ( $65 \%$ of each person's maximum speed). There was an obstacle located in the path of the target requiring the participants to make a curvilinear movement around the object to the target. While healthy participants showed superior retention with a reduced frequency of KR, measured 15 min after the practice phase, people with PD showed more effective retention with $100 \%$ KR. Thus, in contrast to the typical population, it appeared that the reduced KR frequency (20\%) was insufficient to enhance learning in this population, when compared with frequent feedback.

These results are in line with studies (e.g., Contreras-Vidal and Gold, 2004) showing that PD patients have abnormal proprioception, and deficits in the central processing and integration of kinesthetic signals, resulting in an incorrect assembly of multiple sensorimotor inputs into a motor plan. According to these authors, PD patients with degraded kinesthesia, and in the absence of vision of the hand in position matching tasks, must rely on an internal forward model of the relationship between motor commands and their effects in order to obtain real-time estimates of hand and limb position. If the kinesthetic input is degraded, so will be the internal model, with PD patients being unable to store/maintain estimates of limb position derived from proprioceptive feedback alone. This might explain the motor control deficits seen in PD patients and their greater dependency on extrinsic feedback information in attempts to improve and maintain the performance, compared with typical participants.

Yet, knowledge of how extrinsic feedback affects motor learning in people with PD is still limited. Previous studies have used very low frequencies of extrinsic feedback (KR) in comparison with frequent feedback, leaving unanswered the question of how a somewhat reduced KR frequency might affect learning. Furthermore, most of these studies used only immediate rather than delayed retention tests, which may not actually assess relatively permanent changes in behavior (Salmoni et al., 1984).
Given the lack of studies and the importance of this variable for learning, in general, and rehabilitation, in particular, the purpose of the present study was to compare the effects of a reduced frequency of KR in participants with $\mathrm{PD}$. As people with $\mathrm{PD}$ appear to require more feedback than typical participants, we predict that a "slightly" reduced frequency of KR $(66 \%)$ could potentially enhance learning, compared with a $100 \%$ frequency, when measured in a delayed retention test without KR. Different from previous studies, which used very low frequencies of KR (e.g., 20\%) in comparison with frequent feedback, we speculated that a relatively small percentage of trials without KR could give learners the opportunity to process important intrinsic information, enhancing retention when extrinsic feedback is no longer available. In other words, not receiving feedback on a few trials could impose an important challenge for the impaired PD intrinsic feedback system, possibly enabling participants to develop a more accurate internal sense of arm and hand position, while strengthening relationships between motor commands and their effects in the environment. To test this hypothesis, a linear positioning task with a spatial target was used. While the $100 \%$ group received KR after each trial, the $66 \%$ group received KR on two thirds of the trials. To assess learning, a retention test was used one day after the practice phase.

\section{MATERIALS AND METHODS PARTICIPANTS}

Twenty individuals with PD (10 men and 10 women), aged 53-87 years (mean age of the 100\% group: 68.3 years; mean age of the $66 \%$ group: 69.0 years) participated in the study. Only persons in Stages 2 and 3 of the Hoehn and Yahr scale (Goetz et al., 2004) participated in the study. They were optimally medicated for PD and participated in the investigation during the "on" medication cycle. To ensure homogeneity they were divided according to gender (five women in each group), and clinical stage (six participants in stage II and four in stage III in each group). Characteristics of the patients are shown in Table 1. The acquisition and retention phases were carried out at the same time of day on two consecutive days. Informed consent was obtained from the participants and the study was approved by the university's ethics committee. Participants were unaware of the purpose of the experiment, and the task was unfamiliar to all of them.

\section{APPARATUS AND TASK}

A linear positioning task consisting of a straight slide bar, approximately $1 \mathrm{~m}$ in length and fastened to a sturdy base was used (Figure 1). A measuring device secured to the base was used to measure the horizontal displacement of the slide, which was attached to the slide bar. Participants sat with their left shoulder in line with the starting point of the slide. To prevent the use of visual cues, they wore opaque swimming goggles. They were also asked to move the slide and stop it on the target using their right hand (they were all right-handed). In the acquisition and retention phases, the goal was to position the cursor at a distance of $60 \mathrm{~cm}$ from the starting point.

\section{PROCEDURE}

The 20 individuals with PD were randomly assigned to one of two groups, with an equal number of male and female in each group. The $100 \%$ group received KR after each trial, while the $66 \%$ group 
Table 1 | Patients characteristics.

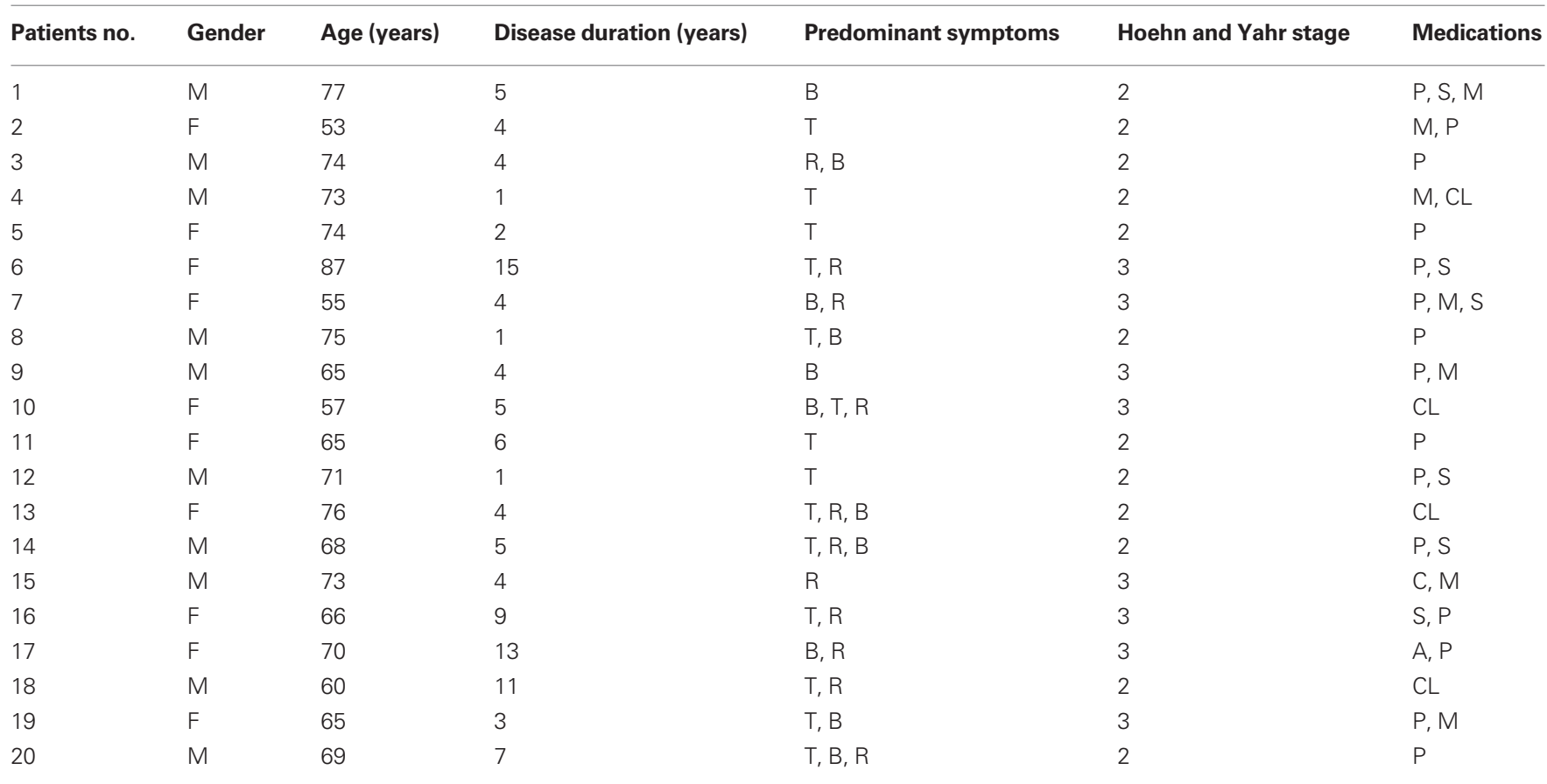

T, resting tremor; $R$, rigidity; $B$, bradykinesia; $P$, prolopa; $M$, mantidan; $S$, sifrol; $C L$, carbidopa + levodopa; $A$, artani.

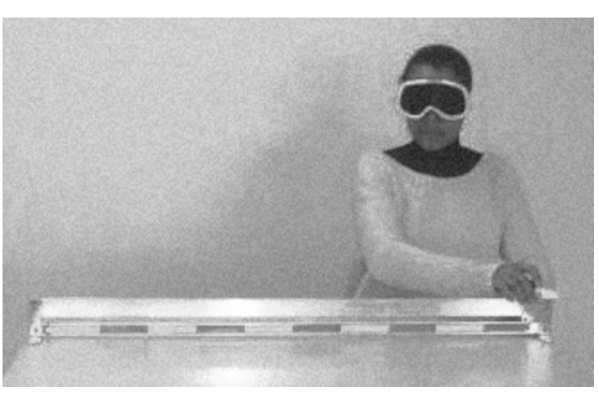

FIGURE 1 | Participant wearing the goggles and positioned in the apparatus.

received KR on two thirds of the trials. More specifically, there were two trials with KR followed by one trial without KR. Participants were asked to sit in front of the apparatus in a comfortable position. The experimenter explained that the objective of the task was to move the slide from its starting position to the right, in one motion, in an attempt to stop it when it reached the target. To prevent visual feedback, participants put on opaque swimming goggles before the beginning of the trials. All participants performed 30 practice trials in the practice phase and 10 trials in the retention phase. The extrinsic feedback informed the participants about the direction (i.e., whether they undershot or overshot the target) and the extent of the deviation from the target in $\mathrm{cm}$ (e.g., -8). After each trial, the experimenter returned the slide to the starting position. No feedback was provided during the retention phase one day after the practice phase.

\section{DATA ANALYSIS}

Constant error (CE), absolute constant error (ACE), and variable error (VE) in cm were our dependent variables. CE is the difference between the actual distance and goal movement distance, representing distance error. ACE is the absolute value of CE for each participant, representing distance error regardless of direction. VE is calculated based on the within-subject variability of the mean for each block of trials, representing a measure of distance consistency (see Schmidt and Lee, 2005 for formula descriptions). Data were averaged across blocks of five trials. The acquisition phase data were analyzed in a 2 (group: 100 versus $66 \% \mathrm{KR}) \times 6$ (blocks of five trials) analysis of variance (ANOVA), with repeated measures on the last factor. Retention data were analyzed in one-way ANOVA. The Greenhouse-Geisser df adjustment was used to report $F$ values in repeated measures factors, if necessary. In order to indicate effect sizes for significant results, partial eta-squared values are reported. Alpha level for significance was set at 0.05 for all analyses.

\section{RESULTS}

\section{PRACTICE}

\section{Constant error}

Both groups tended to undershoot the target on the first block and to overshoot it somewhat on the remaining practice blocks (see Figure 2, left). The main effects of block, $F(5,90)=6.67, p<0.001, \eta^{2}=0.27$, was significant. The main effect of group, $F(1,18)<1$, and the interaction between group and block were not significant, $F(5,90)<1$.

\section{Absolute constant error}

Both groups reduced their absolute errors similarly across practice blocks (see Figure 3, left), particularly on the first three blocks. The main effects of block, $F(5,90)=6.32, p<0.001$, 

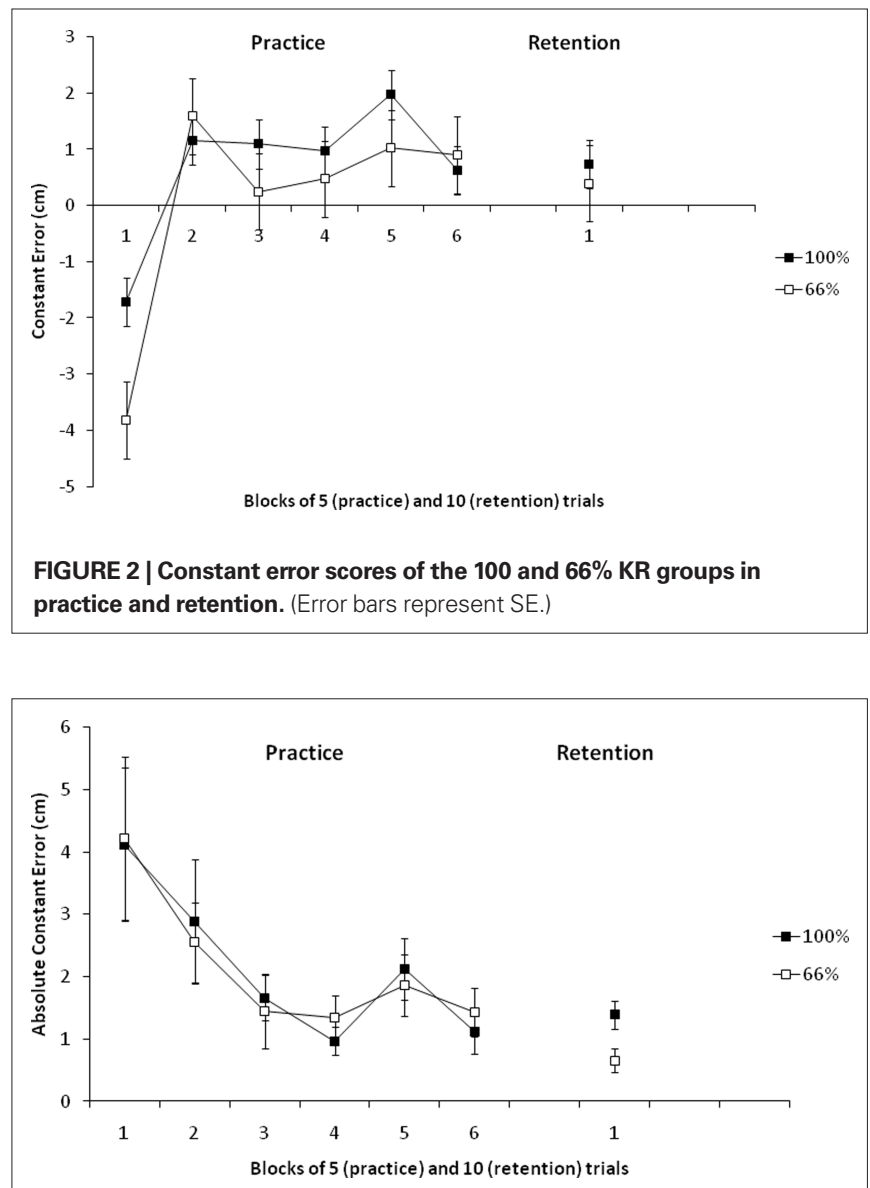

FIGURE 3 | Absolute constant error scores of the 100 and $66 \%$ KR groups in practice and retention. (Error bars represent SE.)

$\eta^{2}=0.26$, was significant. The main effect of group, $F(1,18)<1$, and the interaction between group and block were not significant, $F(5,90)<1$.

\section{Variable error}

Similar to constant and ACEs, both groups reduced their variable errors, with the $66 \% \mathrm{KR}$ group tending to show somewhat larger variability at the beginning of practice, but less variability toward the end (see Figure 4, left). The main effect of block, $F(5,90)=12.47$, $p<0.001, \eta^{2}=0.40$, was significant. However, the main effect of group, $F(1,18)<1$, and the interaction of group and block, $F(5$, $90)=1.14, p>0.05$, were not significant.

\section{RETENTION}

\section{Constant error}

On the retention test without $\mathrm{KR}, 1$ day after practice, the groups demonstrated similar constant errors (see Figure 2, right). The group difference was not significant, with $F(1,19)<1$.

\section{Absolute constant error}

The $66 \%$ KR group had smaller ACEs than the 100\% KR group (see Figure 3, right). The group difference was significant, with $F(1,19)=6.25, p<0.05, \eta^{2}=0.25$.

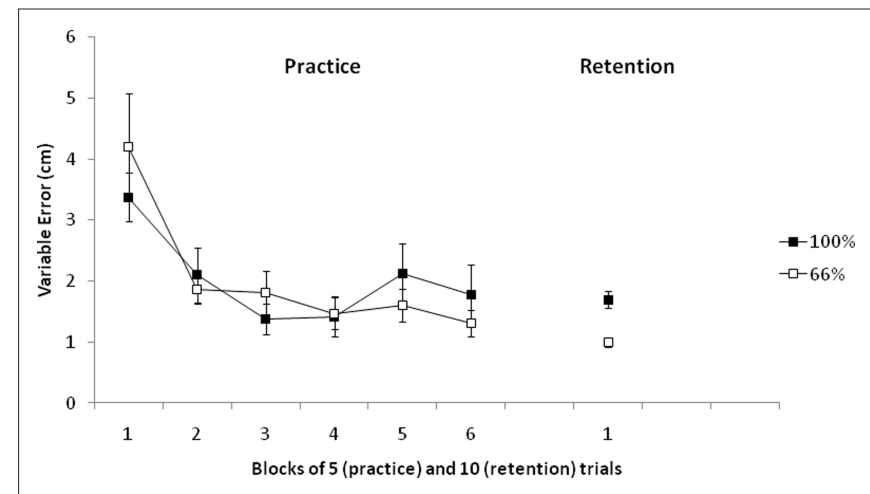

FIGURE 4 | Variable error scores of the 100 and $66 \%$ KR groups in practice and retention. (Error bars represent SE.)

\section{Variable error}

The $66 \%$ KR group showed less variability in performance compared with the $100 \%$ group (see Figure 4, right). The group difference was significant, with $F(1,19)=19.02, p<0.01, \eta^{2}=0.51$.

\section{DISCUSSION}

People with PD differ from typical adults in several ways (Rand et al., 2000; Berardelli et al., 2001; Guadagnoli et al., 2002; Konczak et al., 2007, 2009; Jankovic, 2008; Nieuwboer et al., 2009). Specifically, studies utilizing tasks with spatial goals that demand appropriate use of feedback information, as the linear positioning task used in the present experiment, have demonstrated that people with PD present motor control deficits, resulting, for example, in poorer arm matching accuracy (Rabin et al., 2010) than shown by the typical population. Impairments in the basal ganglia can be responsible for the reduced capability to control and regulate spatial and force parameters. Therefore, individuals with $\mathrm{PD}$ are less able to regulate velocity and acceleration magnitudes when accuracy constraints are imposed (Rand et al., 2000). Together with abnormal proprioception (Adamovich et al., 2001), the deficits in the central processing and integration of kinesthetic signals, resulting in incorrect formulations of motor plans (Contreras-Vidal and Gold, 2004), may explain the greater dependency on extrinsic feedback information in persons with $\mathrm{PD}$.

As previous studies examining feedback frequency effects used very low KR frequencies (Verschueren et al., 1997; Guadagnoli et al., 2002), it was not clear whether a "slightly" reduced KR frequency would enhance the learning of a motor task, compared to feedback after every trial, in this population. Considering that individuals with PD have difficulty in processing intrinsic feedback, with treatment often involving the use of external cues as compensation, the purpose of the present study was to examine whether the learning advantages of a reduced frequency of KR found in typical participants (see Swinnen, 1996; Wulf and Shea, 2004, for reviews) would generalize to motor learning in participants with PD.

Our results are in line with previous studies using typical populations in showing that a reduced frequency of feedback can also benefit the learning of motor skills in participants with PD. According to the guidance hypothesis (Salmoni et al., 1984; Schmidt, 1991), extrinsic feedback guides the learner to the goal 
behavior. However, when provided too frequently, it can cause a dependency, blocking the processing of intrinsic feedback. Also, according to the maladaptive short-term corrections notion, frequent feedback can cause some instability during the practice phase, since the learner makes constant corrections to the action plan, even when errors are small. The result can be less consistent performance in retention and transfer phases. A low frequency of feedback could help the improvement of some stability between trials, resulting in a stronger basis for the use of the feedback information, when provided.

The present results contrast with those of previous studies with PD participants (Verschueren et al., 1997; Guadagnoli et al., 2002), where learning advantages were found for high frequencies of extrinsic feedback. In these studies, severely reduced frequencies of KR were used, leading to inferior learning in comparison with feedback provided after all trials. A possible explanation for the differences in the results could be related to the frequency used in our study. The use of a slightly reduced (66\%) frequency of feedback could have helped participants with PD to rely on intrinsic feedback, despite their difficulties in processing it, during the few trials where the KR was not provided. In this way, the participants were provided with both sufficient extrinsic information and the opportunity to process intrinsic information (on trials without feedback) - enhancing learning and aiding retention in a situation, in which augmented feedback was no longer available.

An alternative interpretation of the present results is that the feedback tended to direct participants' attention to their own movements (i.e., induced an internal focus), which has been shown to be detrimental to learning (Wulf, 2007) - and that frequent feedback (i.e., 100\%) exacerbated this effect. The reduced feedback frequency (i.e., 66\%) may have provided at least some relief from these constant internal focus reminders, resulting in more effective learning (see Wulf et al., 2002, 2010a). Furthermore, the constant error information may have promoted self-related thoughts or concerns, and negatively influenced participants' perception of their capability, which can disrupt movement automaticity and hamper learning (Lewthwaite and Wulf, 2010a,b; Wulf and Lewthwaite, 2010). However, the fact that results of previous studies showed more effective learning with frequent relative to severely reduced frequencies of feedback (e.g., 20\%)

\section{REFERENCES}

Abbruzzese, G., Trompetto, C., and Marinelli, L. (2009). The rationale for motor learning in Parkinson's disease. Eur. J. Phys. Rehabil. Med. 45, 209-214.

Adamovich, S. V., Berkinblit, M. B., Hening, W., Sage, J., and Poizner, H. (2001). The interaction of visual and proprioceptive inputs in pointing to actual and remembered targets in Parkinson's disease. Neuroscience 104, 1027-1041.

Badami, R., VaezMousavi, M., Wulf, G., and Namazizadeh, M. (in press). Feedback after good trials enhances intrinsic motivation. Res. Q. Exerc. Sport.
Berardelli, A. Rothwell, J. C., Thompson, P. D., and Hallett, M. (2001). Pathophysiology of bradykinesia in Parkinson's disease. Brain 124, 2131-2146.

Chiviacowsky, S., and Wulf, G. (2007). Feedback after good trials enhances learning. Res. Q. Exerc. Sport 78, 40-47.

Contreras-Vidal, J. L., and Gold, D. R. (2004). Dynamic estimation of hand position is abnormal in Parkinson's disease. Parkinsonism Relat. Disord. 10, 501-506.

Demirci, M., Grill, S., McShane, L., and Hallett, M. (1997). A mismatch between kinesthetic and visual perception in Parkinson's disease. Ann. Neurol. 41, 781-788.

demonstrates the importance of the informational function of extrinsic feedback for PD patients. Slightly reduced frequencies of feedback may have the benefit of not disrupting automaticity, while providing enough information to enhance learning in this population.

Overall, the present findings demonstrate that reduced frequencies of KR can challenge and enhance learning in people with PD. We conclude that, specifically compared to $100 \% \mathrm{KR}$, a reduced KR frequency of $66 \%$ can result in both greater movement accuracy and movement stability in the learning of a spatial control demand task. To our knowledge, the present study is the first to demonstrate that the benefits of reduced frequencies of KR generalize to motor learning in the PD population. These results may influence the rehabilitation process and procedures used by healthcare professionals working with persons affected by PD in physical activity or exercise-based programs. Using somewhat reduced frequencies of feedback, instructors, or physical therapists may facilitate the learning/relearning of motor skills.

The task used in the present experiment can be considered different from the tasks used in some previous studies (Verschueren et al., 1997; Guadagnoli et al., 2002), since it involved spatial control demands only, without involving temporal aspects. Given that, an important direction for future studies would be to examine whether the benefits of somewhat reduced frequencies of feedback generalize to more complex tasks or situations in people with PD. Since reduced frequencies have the potential to benefit learning in this population, it would also be interesting to examine if feedback provided after effective or "good" trials instead after less effective or "poor" trials (for which benefits have been observed in several recent experiments; Badami et al., in press; Chiviacowsky and Wulf, 2007), would also benefit learning in the PD population. Furthermore, the effects of self-controlled feedback or practice schedules (for a review, see Wulf, 2007) - benefits of which have been shown in typical as well as other special populations - should be examined in participants with PD.

\section{ACKNOWLEDGMENTS}

We thank Dr. Gabriele Wulf and the reviewers for important contributions to the paper. This study received financial support from CNPq (National Council of Scientific and Technological Development), Brazil.

Fisher, B. E., Petzinger, G. M., Nixon, K., Hogg, E., Bremmer, S., Meshul, C. K., and Jakowec, M. W. (2004). Exercise-induced behavioral recovery and neuroplasticity in the 1-methyl4-phenyl- 1,2,3,6-tetrahydropyridine-lesioned mouse basal ganglia. J. Neurosci. Res. 77, 378-390.

Goetz, C. G.,Poewe,W., Rascol,O.,Sampaio, C., Stebbins, G. T., Counsell, C., Giladi, N., Holloway, R. G., Moore, C. G., Wenning, G. K., Yahr, M. D., and Seidl, L. (2004). Movement disorder society task force report on the Hoehn and Yahr staging scale: status and recommendations. Mov. Disord. 19, 1020-1028.

Guadagnoli, M. A., Leis, B., Van Gemmert, A. W., and Stelmach, G.
E. (2002). The relationship between knowledge of results and motor learning in Parkinsonian patients. Parkinsonism Relat. Disord. 9 89-95.

Herman, T., Giladi, N., Gruendlinger, L., and Hausdorff, J. M. (2007). Six weeks of intensive treadmill training improves gait and quality of life in patients with Parkinson's disease: a pilot study. Arch. Phys. Med. Rehabil. 88, 1154-1158.

Hirsch, M. A., and Farley, B. G. (2009). Exercise and neuroplasticity in persons living with Parkinson's disease. Eur. J. Phys. Rehabil. Med. 45, 215-229.

Jankovic, J. (2008). Parkinson's disease: clinical features and diagnosis. 
J. Neurol. Neurosurg. Psychiatry 79, 368-376.

Konczak,J.,Corcos,D.M.,Horak,F., Poizner, H., Shapiro, M., Tuite, P., Volkmann, J., and Maschke,M. (2009).Proprioception and motor control in Parkinson's disease. J. Mot. Behav. 41, 543-552.

Konczak, J., Krawczewski, K., Tuite, P., and Maschke, M. (2007). The perception of passive motion in Parkinson's disease. J. Neurol. 254, 655-663.

Lewthwaite, R., and Wulf, G. (2010a). Grand challenge for movement science and sport psychology: embracing the social-cognitive-affective-motor nature of motor behavior. Front. Psychol. 1:42. doi: 10.3389/ fpsyg.2010.00042

Lewthwaite, R., and Wulf, G. (2010b). Social-comparative feedback affects motor skill learning. Q. J. Exp. Psychol. $63,738-749$.

Maschke, M., Gomez, C. M., Tuite, P. J., and Konczak, J. (2003). Dysfunction of the basal ganglia, but not the cerebellum, impairs kinaesthesia. Brain 126, 2312-2322.

Nieuwboer, A., Rochester, L., Muncks, L., and Swinnen, S. P. (2009). Motor learning in Parkinson's disease: limitations and potential for rehabilitation. Parkinsonism Relat. Disord. 15(Suppl. 3), S53-S58.

Onla-or, S., and Winstein, C. J. (2008). Determining the optimal challenge point for motor skill learning in adults with moderately severe Parkinson's disease. Neurorehabil. Neural Repair 22, 385-395.

Petzinger, G. M., Fisher, B. E., Van Leeuwen, J. E., Vukovic, M., Akopian, G., Meshul, C. K., Holschneider, D. P., Nacca, A., Walsh, J. P., and Jakowec, M. W. (2010).
Enhancing neuroplasticity in the basal ganglia: the role of exercise in Parkinson's disease. Mov. Disord. 25, S141-S145.

Petzinger, G. M., Walsh, J. P., Akopian, G., Hogg, E., Abernathy, A., Arevalo, P., Turnquist, P., Vucković, M., Fisher, B. E., Togasaki, D. M., and Jakowec, M. W. (2007). Effects of treadmill exercise on dopaminergic transmission in the 1-methyl-4-phenyl-1, 2,3,6-tetrahydropyridine-lesioned mouse model of basal ganglia injury. J. Neurosci. 27, 5291-5300.

Rabin, E., Muratori, L., Svokos, K., and Gordon,A. (2010). Tactile/proprioceptive integration during arm localization is intact in individuals with Parkinson's disease. Neurosci. Lett. 5, 470, 38-42.

Rand, M. K., Stelmach, G. E., and Bloedel, J. R. (2000). Movement accuracy constraints in Parkinson's disease patients. Neuropsychologia 38, 203-212.

Salmoni, A. W., Schmidt, R. A., and Walter, C. B. (1984). Knowledge of results and motor learning: a review and critical reappraisal. Psychol. Bull. 95, 355-386.

Schmidt, R. A. (1991). "Frequent augmented feedback can degrade learning: evidence and interpretations," in Tutorials in Motor Neuroscience, eds J. Requin and G. E. Stelmach (Dordrecht: Kluwer Academic Publishers), 59-75.

Schmidt, R.A., and Lee, T.D. (2005). Motor Control and Learning: A Behavioral Emphasis, 4th Edn. Champaign, IL: Human Kinetics.

Swinnen, S. P. (1996). "Information feedback for motor skill learning: a review," in Advances in Motor Learning and Control, ed.H.N.Zelaznik (Champaign, IL: Human Kinetics), 37-66.
Tillerson, J. L., Caudle, W. M., Reveron, M. E., and Miller, G. W. (2003). Exercise induced behavioral recovery and attenuates neurochemical deficits in rodent models of Parkinson's disease. Neuroscience 119, 899-911.

Verschueren, S. M., Swinnen, S. P., Dom, R., and De Weerdt, W. (1997). Interlimb coordination in patients with Parkinson's disease: motor learning deficits and the importance of augmented information feedback. Exp. Brain Res. 113, 497-508.

Winstein, C. J., and Schmidt, R. A. (1990). Reduced frequency of knowledge of results enhances motor skill learning. J. Exp. Psychol. Learn. Mem. Cogn. 16, 677-691.

Wulf, G. (2007). Self-controlled practice enhances motor learning: implications for physiotherapy. Physiotherapy 93, 96-101.

Wulf, G., Chiviacowsky, S., Schiller, E., and Ávila, L. T. G. (2010a). Frequent external-focus feedback enhances motor learning. Front. Psychol. 1:190. doi: 10.3389/fpsyg.2010.00190

Wulf, G., Shea, C., and Lewthwaite, R. (2010b). Motor skill learning and performance: a review of influential factors. Med. Educ. 44: 75-84.

Wulf, G., and Lewthwaite, R. (2010). "Effortless motor learning? An external focus of attention enhances movement effectiveness and efficiency," in Effortless Attention: A New Perspective in Attention and Action, ed. B. Bruya (Cambridge, MA: MIT Press), 75-101.

Wulf, G., McConnel, N., Gärtner, M., and Schwarz, A. (2002). Enhancing the learning of sport skills through external-focus feedback. J. Mot. Behav. 34, 171-182.

Wulf, G., and Schmidt, R. A. (1989). The learning of generalized motor programs: reducing the relative frequency of knowledge of results enhances memory. J. Exp. Psychol. Learn. Mem. Cogn. 15, 748-757.

Wulf, G., and Shea, C. H. (2004). "Understanding the role of augmented feedback: the good, the bad, and the ugly," in Skill Acquisition in Sport: Research, Theory and Practice, eds A. M. Williams and N. J. Hodges (London: Routledge), 121-144.

Conflict of Interest Statement: The authors declare that the research was conducted in the absence of any commercial or financial relationships that could be construed as a potential conflict of interest.

Received: 18 September 2010; accepted: 29 November 2010; published online: 16 December 2010.

Citation: Chiviacowsky S, Campos T and Domingues MR (2010) Reduced frequency of knowledge of results enhances learning in persons with Parkinson's disease. Front. Psychology 1:226. doi: 10.3389/ fpsyg.2010.00226

This article was submitted to Frontiers in Movement Science and Sport Psychology, a specialty of Frontiers in Psychology.

Copyright () 2010 Chiviacowsky, Campos and Domingues. This is an open-access article subject to an exclusive license agreement between the authors and the Frontiers Research Foundation, which permits unrestricted use, distribution, and reproduction in any medium, provided the original authors and source are credited. 